\title{
Dopant stability and strain states in Co and Mn-doped Ge (001) epitaxial films
}

\author{
B. A. Collins, ${ }^{1}$ Y. S. Chu,${ }^{2}$ L. He, ${ }^{1}$ Y. Zhong, ${ }^{2}$ and F. Tsui ${ }^{1, *}$ \\ ${ }^{1}$ Department of Physics and Astronomy, University of North Carolina, Chapel Hill, North Carolina 27599, USA \\ ${ }^{2}$ Advanced Photon Source, Argonne National Laboratory, Argonne, Illinois 60439, USA
}

(Received 19 November 2007; revised manuscript received 25 January 2008; published 1 May 2008)

\begin{abstract}
Systematic investigation of structural, chemical, and magnetic properties of Co and Mn-doped Ge (001) as a function of doping concentration reveals that codoping with Co can dramatically reduce phase separation and diffusion of $\mathrm{Mn}$ within the Ge lattice while it magnetically complements $\mathrm{Mn}$. The measured strain states indicate the critical role played by substitutional Co with its strong tendency to dimerize with interstitial Mn. Selecting appropriate codopants that form energetically stable dimers in a semiconductor host is shown to be a viable approach, thus demonstrating the feasibility for engineering stable doped magnetic semiconductors.
\end{abstract}

DOI: 10.1103/PhysRevB.77.193301

PACS number(s): 68.55.-a, 68.35.-p, 64.75.- $\mathrm{g}$

One of the critical challenges for the science and technology of spintronics is the ability to control synthesis and properties of doped magnetic semiconductors (DMSs). Studies of $\mathrm{DMSs}^{1,2}$ have shown that carrier-mediated ferromagnetism can be enhanced by increasing the doping concentration of a transition metal into a semiconductor matrix, directly influencing both carriers and magnetic ions. Previous efforts to increase doping concentration aimed at realizing room temperature ferromagnetism in DMSs, however, have been hampered by the very low solubility of transition metal species with a strong tendency to form metallic clusters ${ }^{3,4}$ or heterogeneous regions ${ }^{5-7}$ within the matrix. Recent studies of a doped II-VI compound ${ }^{8}$ show that introducing a nonmetallic codopant can suppress not only the tendency to form dopantrich clusters, owing to a change in the charge states of the dopants, but also perhaps undesirably the ferromagnetism associated with the clusters. Recent publications ${ }^{9-12}$ on $\mathrm{Ge}$ based DMSs suggest that codoping with multiple transition metal dopants may alter energetics and kinetics of epitaxial growth, thus enhancing both dopant stability and magnetic properties. Here, we report direct evidence of enhanced stability of transition metal codopants in a DMS epitaxial film. Systematic investigation as a function of doping concentration reveals that codoping with Co can dramatically reduce phase separation and diffusion of $\mathrm{Mn}$ within the Ge lattice while it magnetically complements $\mathrm{Mn}$.

The composition-dependent experiments for the Ge-based ternary epitaxial system were carried out by the use of combinatorial approaches. The ternary composition spread sample $\mathrm{Co}_{x} \mathrm{Mn}_{y} \mathrm{Ge}_{1-x-y}$ (001) was grown by combinatorial molecular beam epitaxy (MBE) techniques ${ }^{13,14}$ on a Ge (001) substrate at $250{ }^{\circ} \mathrm{C}$ to a thickness of $48 \mathrm{~nm}$ and annealed at $450{ }^{\circ} \mathrm{C}$. Prior to the growth of the doped film, a Ge buffer layer of $\sim 40 \mathrm{~nm}$ was grown and annealed. The lateral dimension of the triangular ternary region of the sample was approximately $5 \mathrm{~mm}$ across. Additional samples were also grown under these conditions for magnetic and magnetotransport measurements.

Structure and composition of the sample were examined by using microbeam x-ray diffraction (XRD) and fluorescence (XRF) techniques at the 2-BM beamline of the Advanced Photon Source (APS) at Argonne National Laboratory. ${ }^{14,15}$ Depth-dependent composition profiles and dopant diffusion were examined by dynamic secondary ion mass spectrometry (SIMS) experiments. SIMS yields for Co and $\mathrm{Mn}$ in Ge were calibrated using standards with comparable doping concentrations. Crosshairs with a linewidth of $\sim 10 \mu \mathrm{m}$ were scribed on the sample and used to correlate the composition of various measurements, resulting in a compositional reproducibility of better than 0.2 at. \% . Magnetic and magnetotransport measurements were carried out using superconducting quantum interference device magnetometry and four-terminal techniques, respectively.

The ternary epitaxial film at low doping concentrations is pseudomorphic and atomically smooth, as determined by the off-specular XRD measurements. ${ }^{11,15}$ Beyond a critical composition, rough disordered growth occurs, exhibiting two primary disorders: stacking faults and polycrystalline secondary phases, as shown in Fig. 1. In the Co-rich regions of composition, XRD measurements ${ }^{14}$ reveal the formation of stacking faults [Fig. 1(a)] with no detectable secondary phases. Intensities from stacking faults are from the twinned $\{022\}$ reflections. In contrast, in the Mn-rich regions, especially along $\mathrm{Mn}_{y} \mathrm{Ge}_{1-y}$, increasing doping concentration leads to precipi-

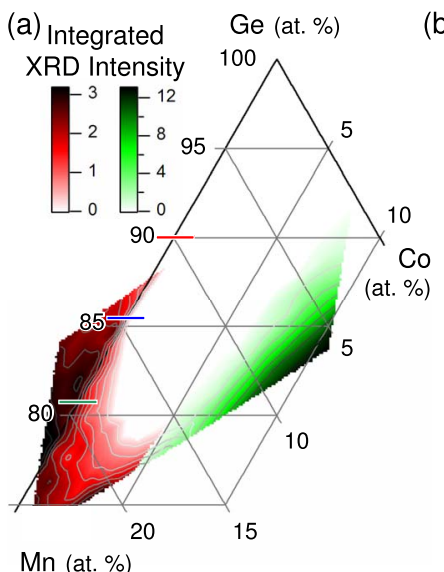

$\mathrm{Mn}$ (at. \%)

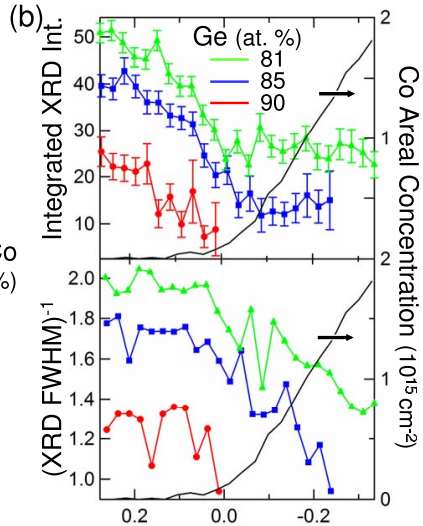

Position on Sample (mm)
FIG. 1. (Color online) Composition-dependent evolution of disorders in Co and Mn codoped Ge (001) epitaxial film. (a) Integrated XRD intensities of secondary phases shown in red (dark gray) and the stacking faults along $\langle 111\rangle$ directions (green): the populations of the disorders as a function of composition. (b) Integrated XRD intensity (top) and inverse of XRD full width at half maximum (bottom) of the secondary phases versus Co concentration. 

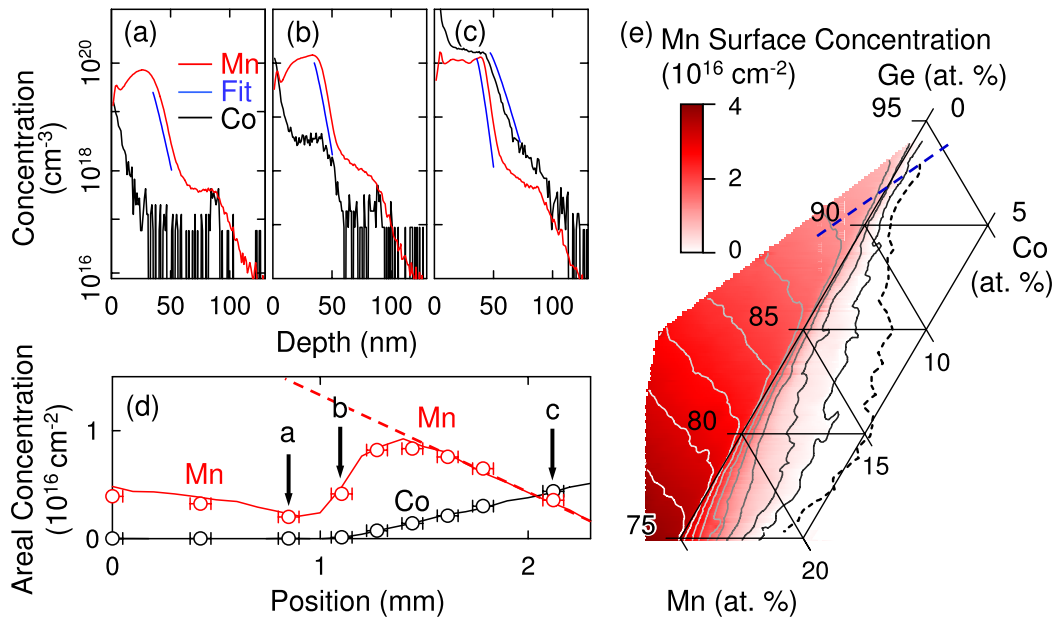

FIG. 2. (Color online) Depth dependent Mn and Co concentrations for $\mathrm{Co}_{x} \mathrm{Mn}_{y} \mathrm{Ge}_{1-x-y}$ (001). Dynamic SIMS profiles in Ge (a) without $\mathrm{Co}$, (b) trace amount of Co $\left(\sim 10^{14} \mathrm{~cm}^{-2}\right)$, and (c) few at. \% Co. The blue (dark gray) lines correspond to exp $\left(-\alpha z^{n}\right)$ fits with respective $n$ of $1.10,1.16,1.22$, and 1.17, and for clarity, they are horizontally shifted. (d) Evolution of dopants within the film versus position of the sample, as determined by integrating the SIMS yields (circles) and by XRF measurements (lines). The dashed line corresponds to XRF measurements before removal of the surface Mn, whereas solid lines correspond to those after the treatment. The arrows and labels indicate the respective locations of the depth profiles shown above. (e) Areal concentration of surface Mn determined by XRF measurements before and after removal of the surface aggregates, i.e., the difference between the two measured concentrations. The contours are in steps of 5 $\times 10^{15} \mathrm{~cm}^{-2}$ except for the last two, with the dotted curve equivalent of one atomic layer, and the second to last about three atomic layers $\left(2 \times 10^{15} \mathrm{~cm}^{-2}\right)$. The blue (dark gray) dashed line indicates the location of the results shown in (d).

tation of secondary phases, identified as textured polycrystalline $\mathrm{Mn}$ and $\mathrm{MnO}_{2}$ precipitates [Fig. 1(a)] without any detectable stacking faults.

As shown in Fig. 1, the onset of Co doping gives rise to a significant reduction in the intensities associated with the Mn secondary phases, accompanied by a broadening of the XRD peak widths, which is likely to be the result of a reduction in the average grain size of the Mn precipitates. The observed behavior provides direct evidence for reducing and suppressing $\mathrm{Mn}$ precipitation in Ge using Co as a codopant and thus demonstrates that Mn ions can be stabilized in a Ge matrix by Co.

In Fig. 2, dopant concentrations and their depth dependence provide the critical evidence for Mn stabilization in $\mathrm{Ge}$ versus Co concentration. Beneath a transient surface layer of $\sim 10 \mathrm{~nm},{ }^{16}$ each SIMS depth profile exhibits three distinct regions: the doped film, the Ge buffer, ${ }^{17}$ and the Ge substrate, which are separated by exponential decays. In the absence of Co doping [Fig. 2(a)], the Mn depth profile within the film exhibits a rounded shape with an integrated amount of $\mathrm{Mn}$ that is significantly lower than what was deposited [Fig. 2(d)]. This indicates a depletion of Mn dopants to the surface, which is consistent with recent studies. ${ }^{18-20}$ However, trace amounts of Co have led to a flattening of the Mn profile in the film with a well-defined interface [Fig. 2(b)] and a corresponding sharp increase in the amount of $\mathrm{Mn}$ remaining in the film [Fig. 2(d)]. As Co concentration increases, the $\mathrm{Mn}$ concentration rapidly reaches what was deposited [the dotted line in Fig. 2(d)], and its depth profile becomes constant within the film, which is accompanied by a sharp exponential drop at the film-buffer interface [Fig. 2(c)].

Since $\mathrm{Mn}$ is very brittle and slowly decomposes in water, excessive sonication in organic solvents was used to remove the surface aggregate without damaging the film underneath.
The removal of the surface Mn precipitates was confirmed by XRD and XRF measurements, in addition to electron energy dispersive $\mathrm{X}$-ray spectroscopy and optical reflectivity measurements. ${ }^{14}$ In Fig. 2(d), XRF results for Mn before and after removing the surface $\mathrm{Mn}$ are compared to the integrated SIMS yields (circles), taken along the same line on the sample [the dashed line in Fig. 2(e)]. The excellent agreement between the latter two indicates that the XRF results after removing the surface $\mathrm{Mn}$ are the Mn concentration dispersed into the film. In contrast, Co is fully dispersed in the film, and removing the surface precipitates does not appear to affect the film beneath. Most importantly, the concentration of surface Mn, as shown in Fig. 2(e), exhibits an exponential decrease as $\mathrm{Co}$ is introduced as a codopant, leading ultimately to the suppression of surface precipitation and stable $\mathrm{Mn}$ in the film.

The SIMS depth profiles also provide insight into the diffusion processes, since the decay rate below the film-buffer interface is a direct measure of diffusion rate. The increase in slope from Figs. 2(a)-2(c) indicates that the presence of $\mathrm{Co}$ significantly limits the diffusion of Mn. Furthermore, studies $^{21}$ show that the functional form on depth $(z)$ depends on the diffusion mechanism, such that single channel interstitial diffusion via Fick's law gives rise to an asymptotic exponential dependence on $z^{2}$, whereas multichannel diffusion in the presence of traps yields a corresponding dependence on $z$. The best fits of the depth profiles yield exponents of $z$ that are between 1 and 1.2 [Figs. 2(a)-2(c)] rather than 2 , indicating multichannel diffusion as the dominant mechanism. As discussed below, the likely diffusion traps are substitutional $\mathrm{Co}$ that tends to attract interstitial $\mathrm{Mn}$ to form a stable dimer.

Strain states and crystalline quality of the epitaxial film as a function of composition have been examined by using 
XRD measurements and X-ray crystal truncation rod analysis, ${ }^{14,15}$ as shown in Fig. 3. The XRD patterns in regions I and II [Fig. 3(a)] can be best described as those from two-dimensional coherent epitaxial films, ${ }^{15}$ while those in regions III and IV instead correspond to inhomogeneous and rough epitaxial films. The strain values (Fig. 3) (defined as $\varepsilon=a_{f} / a_{s}-1$ with $a_{f}$ and $a_{s}$ as the respective lattice parameters of the film and the substrate) have been obtained by using a simple kinematical model, and they correspond to the lattice mismatch between the film and the Ge substrate after correcting for the elastic distortion due to coherent epitaxy. $^{10,15}$

Within regions of high quality coherent epitaxy, the film strains $\varepsilon$ can be characterized by two planes: one Co rich (I) and another Mn rich (II). Each plane can be fit to Vegard's law given by $\varepsilon=\varepsilon_{\mathrm{Co}_{0}} x+\varepsilon_{\mathrm{Mn}} y$, where the variables $x$ and $y$ are the respective doping concentrations of $\mathrm{Co}$ and $\mathrm{Mn}$ with the corresponding strain coefficients $\varepsilon_{\mathrm{Co}}$ and $\varepsilon_{\mathrm{Mn}}$, as they are listed in Table I. Nearly the entire region I [Fig. 3(a)] can be fit to a single plane, and in contrast to region II, only the counterpart at low Mn concentrations fits to a plane. However, upon close examination, even region I contains a second linear component at higher doping concentrations, as

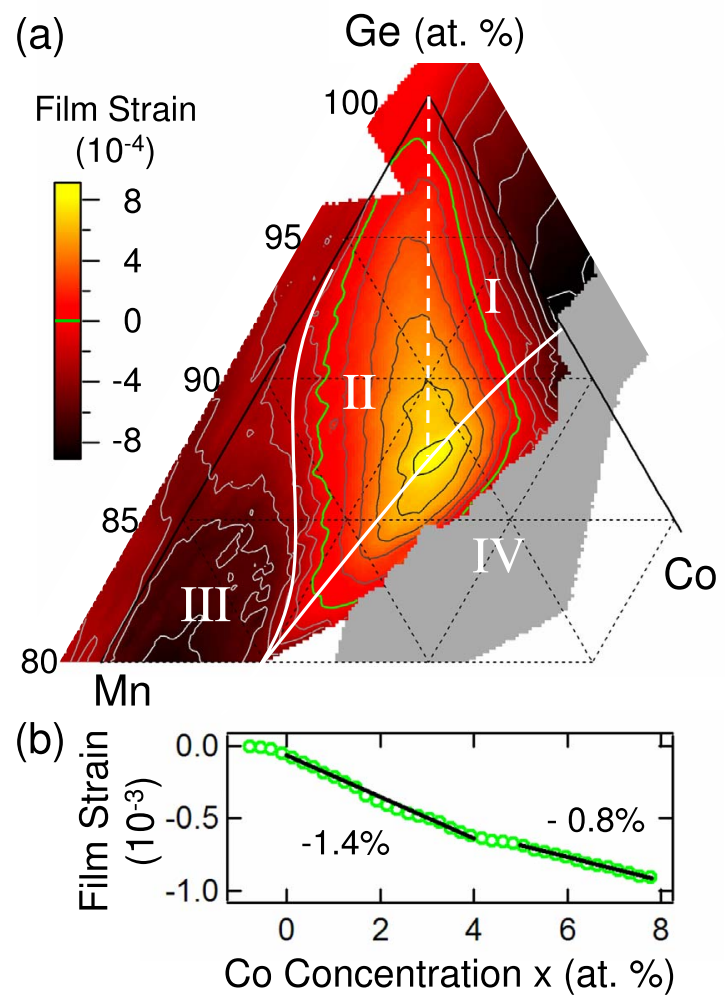

FIG. 3. (Color online) (a) Film strain as a function of composition for ternary $\mathrm{Co}_{x} \mathrm{Mn}_{y} \mathrm{Ge}_{1-x-y}(001)$ epitaxial thin film and regions with different structural characteristics (separated by white lines). The interval between the contour lines of strain is $0.015 \%$. Regions I and II are free of detectable disorders. The film contains Mn surface aggregation in region III and stacking faults in region IV, with the gray area indicating the region of rough films. (b) Film strain (circles) for binary $\mathrm{Co}_{x} \mathrm{Ge}_{1-x}$ with linear fits (lines and slopes).
TABLE I. Vegard's law coefficients for the coherent regions [I and II in Fig. 3(a)] of ternary $\mathrm{Co}_{x} \mathrm{Mn}_{y} \mathrm{Ge}_{1-x-y}$ (001) epitaxial film, which are obtained from fitting the film strains to $\varepsilon(x, y)=\varepsilon_{\mathrm{Co}} x$ $+\varepsilon_{\mathrm{Mn}} y$. The uncertainties correspond to variations in the fits made from selecting different compositional ranges.

\begin{tabular}{lcc}
\hline \hline Region of composition & $\begin{array}{c}\varepsilon_{\mathrm{Co}} \\
(\%)\end{array}$ & $\begin{array}{c}\varepsilon_{\mathrm{Mn}} \\
(\%)\end{array}$ \\
\hline Region I & $-0.9 \pm 0.1$ & $+2.8 \pm 0.1$ \\
Region II for $y<8$ at. $\%$ & $1.4 \pm 0.1$ & $-0.5 \pm 0.1$ \\
Region II for $y>8$ at. $\%$ & $1.4 \pm 0.1$ & $-1.2 \pm 0.1$ \\
\hline \hline
\end{tabular}

shown in Fig. 3(b). The observed linear dependence of strains is a strong indication that the dopants are dispersed into the lattice.

The coefficients in Vegard's law are often directly interpreted as the difference in average atomic size between the dopant and the host matrix. However, the presence of two planes and their symmetry suggest that there are at least three species of dopants. In what follows, a likely atomistic mechanism is put forward for the stable dopant species, which is based on the evidence presented here and the energetics of the system.

Recent calculations ${ }^{12}$ show that certain types of dimers are energetically more favorable than isolated substitutional monomers. Specifically, dimers with $\mathrm{Co}$ at a substitutional site and $\mathrm{Mn}$ at an adjacent interstitial site are by far the most stable, reducing energy by more than $1 \mathrm{eV} /$ atom over those of the monomers. The stable substitutional Co evidently plays the role of attracting mobile interstitial $\mathrm{Mn}$ to form the stable dimer, which in turn blocks a diffusion channel. This reduces the diffusion of other dopants and limits the population of larger clusters at finite temperatures, which agrees with the observed substantial effect that Co has on Mn even at trace amounts [Fig. 2(b)]. Therefore, by forming Co-Mn dimers, Co acts as a trap for the otherwise rapidly diffusing Mn interstitials and alters the diffusion process, as discussed above (Fig. 2).

This mechanism would result in three main populations of species that contribute to the epitaxial strain at low concentrations, Co-Mn dimers of the type described above, substitutional $\mathrm{Co}$ in the Co-rich region (I), and substitutional $\mathrm{Mn}$ in the Mn-rich region (II). A modified Vegard's law that includes all three species is necessary in order to describe the measured strains. For region I, strain depends on the concentration of Co-Mn dimers and on that of the excess Co, i.e., $\varepsilon=\varepsilon_{\mathrm{Co}}(x-y)+\varepsilon_{\mathrm{Co}-\mathrm{Mn}} y$, and correspondingly for region II, $\varepsilon$ $=\varepsilon_{\mathrm{Co}-\mathrm{Mn}} x+\varepsilon_{\mathrm{Mn}}(y-x)$, where the coefficient $\varepsilon_{\mathrm{Co}-\mathrm{Mn}}$ is associated with the size of the Co-Mn dimer. The symmetry of the boundary between the two regions (along Co to $\mathrm{Mn}$ atomic ratio of 1:1) is also consistent with this interpretation. Consequently, the values of atomic radii can be obtained by using the above definition of strain, and they are $0.121 \mathrm{~nm}$ for substitutional Co, $0.126 \mathrm{~nm}$ for interstitial $\mathrm{Mn}$ associated with the dimer, and $0.122 \mathrm{~nm}$ for substitutional Mn by using the Ge value of $0.122 \mathrm{~nm}$. These values are in excellent agreement with the first principles calculations. ${ }^{12}$ At higher doping concentrations, other populations of species may be- 

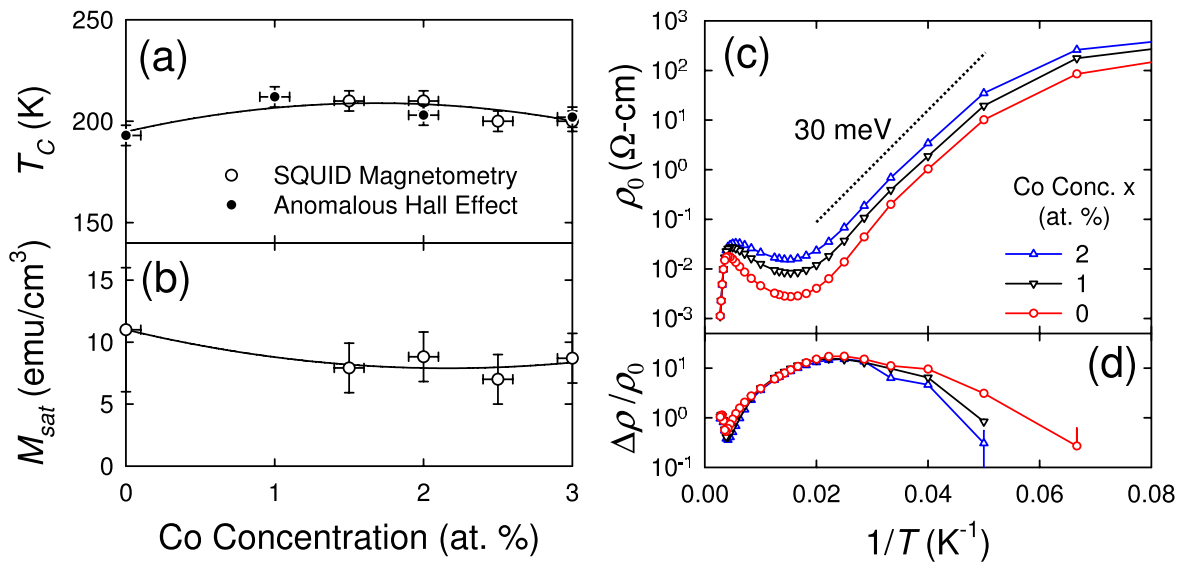

FIG. 4. (Color online) Magnetic and magnetotransport properties of $\mathrm{Co}_{x} \mathrm{Mn}_{0.04-x} \mathrm{Ge}_{0.96}$ (001) epitaxial films versus Co concentration $x$. (a) Curie temperature and (b) saturation magnetization at $5 \mathrm{~K}$ versus $x$. (c) Resistivity in zero field and (d) magnetoresistance at $5 \mathrm{~T}\left[\equiv \rho(H=5 \mathrm{~T}) / \rho_{0}-1\right]$ versus inverse temperature for various $x$. The behaviors for $x$ $\geqslant 2$ at. $\%$ are nearly identical. Lines are guides for the eyes. come energetically viable, including interstitial Co [Fig. 3(b)].

Finally, the question of whether codoping with Co is magnetically complementary to Mn doping in Ge has been examined using samples with a constant Ge concentration of 96 at. \%. Our magnetic and magnetotransport measurements show that these samples are $p$-type ferromagnetic semiconductors with a very large positive magnetoresistance $(>10$ at $5 \mathrm{~T}$ ) and very large anomalous Hall coefficients, consistent with an earlier report for the system. ${ }^{9}$ As shown in Fig. 4, substituting Mn with Co does not significantly affect magnetic and magnetotransport parameters. Based on the mechanism described above, replacing $\mathrm{Mn}$ with Co-Mn dimers appears to slightly enhance the Curie temperature [Fig. 4(a)] and reduce the saturation magnetization $M_{\text {sat }}[$ Fig. 4(b)]. The reduction in $M_{\text {sat }}$ corresponds to a change in the average moment per dopant ion from $\sim 0.5 \mu_{B}$ at $x=0$ to $\sim 0.3 \mu_{B}$. The resistivity in the $p$-type extrinsic regime also exhibits an increase as Co concentration increases [Fig. 4(c)], which is accompanied by a corresponding decrease in the hole concentration (estimated from the ordinary Hall effect) from 1 $\times 10^{17} \mathrm{~cm}^{-3}$ at $x=0$ to $6 \times 10^{16} \mathrm{~cm}^{-3}$ at $x=1$ at. $\%$ and to 4 $\times 10^{16} \mathrm{~cm}^{-3}$ for $x \geq 2$ at. $\%$. This indicates that $\mathrm{Mn}$ as a dop- ant is slightly more effective than Co in contributing to the hole concentration and to the magnetization in Ge, which is consistent with the theoretical work. ${ }^{12}$ Furthermore, the impurity states associated with the two dopants appear to be comparable to each other, as indicated by the nearly identical temperature dependence and activation energies for the transport parameters at low temperature [the dotted line in Fig. 4(c)]. These findings indicate that as codopants in Ge, $\mathrm{Co}$ and $\mathrm{Mn}$ are complementary both magnetically and electronically.

The authors wish to thank S. Vogt and Q. Shen for discussion and support. The work was supported in part by U.S. Department of Energy, Office of Sciences, Office of Basic Energy Sciences Grant No. DE-FG02-05ER46216, by U.S. NSF Grant No. DMR-0441218 for MBE synthesis, and by U.S. DOD Grant No. W911NF-05-1-0173 for MBE instrumentation. Use of the Advanced Photon Source is supported by the U.S. Department of Energy, Office of Sciences, Office of Basic Energy Sciences, under Contract No. DE-AC0206CH11357. An APS under Contract No. 5F-00428 for partial student support is also acknowledged. *ftsui@physics.unc.edu

${ }^{1}$ H. Ohno, Science 281, 951 (2002).

${ }^{2}$ T. Dietl, H. Ohno, and F. Matsukura, Phys. Rev. B 63, 195205 (2001).

${ }^{3}$ J. S. Kang et al., Phys. Rev. Lett. 94, 147202 (2005).

${ }^{4}$ L. Ottaviano et al., Appl. Phys. Lett. 88, 061907 (2006).

${ }^{5}$ M. Jamet et al., Nat. Mater. 5, 653 (2006).

${ }^{6}$ A. P. Li, C. Zeng, K. van Benthem, M. F. Chisholm, J. Shen, S. V. S. Nageswara Rao, S. K. Dixit, L. C. Feldman, A. G. Petukhov, M. Foygel, and H. H. Weitering, Phys. Rev. B 75, 201201(R) (2007).

${ }^{7}$ D. Bougeard, S. Ahlers, A. Trampert, N. Sircar, and G. Abstreiter, Phys. Rev. Lett. 97, 237202 (2006).

${ }^{8}$ T. Dietl, Nat. Mater. 5, 673 (2006); S. Kuroda et al., ibid. 6, 440 (2007)

${ }^{9}$ F. Tsui, L. He, L. Ma, A. Tkachuk, Y. S. Chu, K. Nakajima, and T. Chikyow, Phys. Rev. Lett. 91, 177203 (2003).

${ }^{10}$ F. Tsui, L. He, A. Tkachuk, S. Vogt, and Y. S. Chu, Phys. Rev. B 69, 081304(R) (2004).
${ }^{11}$ L. He, B. A. Collins, F. Tsui, Y. Zhong, S. Vogt, and Y. S. Chu, J. Vac. Sci. Technol. B 25, 1217 (2007).

${ }^{12}$ A. Continenza, G. Profeta, and S. Picozzi, Phys. Rev. B 73, 035212 (2006); Appl. Phys. Lett. 89, 202510 (2006).

${ }^{13}$ F. Tsui and L. He, Rev. Sci. Instrum. 76, 062206 (2005).

${ }^{14}$ F. Tsui et al., Appl. Surf. Sci. 254, 709 (2007).

${ }^{15}$ Y. Zhong, Y. S. Chu, B. A. Collins, and F. Tsui, Appl. Surf. Sci. 254, 714 (2007).

${ }^{16}$ The near surface concentrations cannot be determined by dynamic SIMS, owing to initial ion beam damage (in this case, $\sim 10 \mathrm{~nm}$ ).

${ }^{17} \mathrm{Mn}$ contamination of $\sim 10^{17} \mathrm{~cm}^{-3}$ during MBE growth of the Ge buffer.

${ }^{18}$ K. W. Edmonds et al., Phys. Rev. Lett. 92, 037201 (2004).

${ }^{19}$ P. Gambardella et al., Phys. Rev. B 75, 125211 (2007).

${ }^{20}$ W. Zhu, H. H. Weitering, E. G. Wang, E. Kaxiras, and Z. Zhang, Phys. Rev. Lett. 93, 126102 (2004).

${ }^{21}$ L. Y. Wei, J. Phys. Chem. Solids 18, 162 (1961). 